\title{
Ketamine exerts antidepressant effects and reduces IL-1ß and IL-6 levels in rat prefrontal cortex and hippocampus
}

\author{
CHUN YANG ${ }^{1}$, TAO HONG $^{1}$, JIANG SHEN $^{1}$, JIE DING $^{1}$, XIONG-WEI DAI ${ }^{1}$, \\ ZHI-QIANG ZHOU ${ }^{2}$ and JIAN-JUN YANG ${ }^{2}$ \\ ${ }^{1}$ Department of Anesthesiology, The Third Affiliated Hospital of Suzhou University, Changzhou; \\ ${ }^{2}$ Department of Anesthesiology, Jinling Hospital, School of Medicine, Nanjing University, Nanjing, P.R. China
}

Received November 28, 2012; Accepted January 21, 2013

DOI: $10.3892 /$ etm.2013.930

\begin{abstract}
Ketamine has fast-acting and robust antidepressant effects in animal models and depressed patients. It has been hypothesized that its underlying mechanism of action is associated with the inflammatory response in the central nervous system. Therefore, the present study was designed to investigate the antidepressant effects of ketamine and the expression of interleukin (IL)- $1 \beta$ and IL- 6 in the prefrontal cortex and hippocampus of a rat model. Twenty Wistar rats were randomly divided into 2 groups (each group, $n=10$ ); the saline group and the ketamine group. On the 1st day, rats undertook a forced swimming test (FST) for $15 \mathrm{~min}$ (pre-test session). On the 2nd day, saline or ketamine was administered intraperitoneally $30 \mathrm{~min}$ before the test session. Following this, rats performed another FST for 5 min (test session) and the immobility time was recorded. The rats were then sacrificed, and the prefrontal cortex and hippocampus were harvested for determination of IL-1 $\beta$ and IL- 6 levels. Compared with the saline group, ketamine administration significantly decreased the immobility time of rats during the FST $(\mathrm{P}<0.05)$. In addition, the ketamine group demonstrated a statistically significant decrease in the expression of IL-1 $\beta$ and IL-6 in rat prefrontal cortex and hippocampus compared with the saline group $(\mathrm{P}<0.05)$. Ketamine-induced antidepressant effects are associated with decreased levels of IL-1 $\beta$ and IL-6 in rat prefrontal cortex and hippocampus.
\end{abstract}

\section{Introduction}

Depression is a debilitating disorder with severe health problems which pose a heavy economic burden to society (1). At present, monoamine drugs are the dominant therapeutic agent of choice for the treatment of depression (2). However, currently

Correspondence to: Professor Jian-Jun Yang, Department of Anesthesiology, Jinling Hospital, School of Medicine, Nanjing University, 305 East Zhongshan Road, Nanjing 210002, P.R. China

E-mail: hippocampus@sohu.com

Key words: ketamine, antidepressant effects, interleukin-1 $\beta$, interleukin-6, prefrontal cortex, hippocampus available antidepressant agents have severe drawbacks, such as low rates of treatment response and delayed onset time $(3,4)$. In several studies, depression is considered as the disease that is related to the high expression of proinflammatory cytokines, and furthermore, attenuation of inflammation is suggested as one possible mechanism explaining the action of antidepressant agents (5-7). Collectively, the inflammatory response system is likely to be implicated in the etiology and treatment of depression.

Ketamine, an ionotropic glutamatergic N-methyl-D-aspartic acid (NMDA) receptor antagonist, is widely used in clinical anesthesia and pain treatment. Recent clinical studies have provided evidence that sub-anesthetic doses of ketamine may rapidly alleviate symptoms of depression, even for treatmentresistant depression, with an identical therapeutic effect $(8,9)$. However, its underlying mechanisms have yet to be fully elucidated.

A previous review summarized that ketamine has the potential to modulate inflammation (10). It has been widely acknowledged that ketamine has been recommended for use in the surgery of sepsis patients due to its anti-inflammatory effects $(11,12)$. However, too little attention is presently focused on the underlying mechanism with regard to whether inflammatory cytokines are involved in the antidepressant effects of ketamine.

Thus, we propose a hypothesis that ketamine may exert antidepressant effects via modulation of proinflammatory cytokines. Based on this theory, we aimed to validate the above-mentioned hypothesis. The present study was designed to determine the levels of IL- $1 \beta$ and IL- 6 in the rat prefrontal cortex and hippocampus after ketamine administration during a forced swimming test (FST).

\section{Materials and methods}

Animals and drugs. Twenty male Wistar rats weighing 180-300 g were purchased from the Shanghai Animal Center (Shanghai, China). Five rats were housed per cage with food and water available ad libitum and maintained on a 12-h light/dark cycle (lights on at 07:00 am). Rats were randomly divided into 2 groups (each group, $n=10$ ). Rats were intraperitoneally administered with saline or ketamine at a dose of $10 \mathrm{mg} / \mathrm{kg} 30 \mathrm{~min}$ before the test session of FST. Ketamine was 
obtained from the Gutian Pharmaceutical Company (Fujian, China). The experimental procedures were approved by the Institutional Animal Ethics Committee of Suzhou University (Suzhou, China).

FST. FST was applied according to our previous study (13) to evaluate the antidepressant effects of ketamine. The test included two separate sessions in a cylindrical tank $(30-\mathrm{cm}$ diameter, $60-\mathrm{cm}$ height) filled with water $\left(22-23^{\circ} \mathrm{C}\right)$ to a $30-\mathrm{cm}$ level in which rats were unable to touch the bottom of the tank. The water was replaced after each rat had completed one session. All procedures were conducted between 9:00 am-15:00 pm. Rats first underwent a FST for 15 min (pre-test session). After $24 \mathrm{~h}$, rats were placed in the water again for $5 \mathrm{~min}$ (test session), and the immobility time was recorded in seconds. Immobility was defined as the amount of time that the rat remained floating in the water without struggling and made only those movements necessary to keep its head above the water.

Testing IL-1 $\beta$ levels. Following FST, rats were immediately sacrificed and the prefrontal cortex and hippocampus were harvested for determination. In brief, the prefrontal cortex and hippocampus were separately washed with ice-cold phosphate-buffered solution (PBS) and scraped in lysis buffer. The insoluble material was removed by centrifugation at $12,000 \mathrm{rpm}$ for $20 \mathrm{~min}$. Protein content was measured according to the bicinchoninic acid (BCA) method. Non-specific binding was blocked for $1 \mathrm{~h}$ at $37^{\circ} \mathrm{C}$ in TBS containing non-fat dried milk. The membrane was then incubated with primary antibodies against IL-1 $\beta(1: 1000)$ and $\beta$-actin served as the control. Membranes were then incubated in the appropriate HRP-conjugated secondary antibodies (1:20000).

Testing IL-6 levels. IL-6 levels in the prefrontal cortex and hippocampus were measured by sandwich-ELISA of relevant primary antibodies according to the manufacturer's instructions (Chemicon, Temecula, CA, USA). Briefly, rat prefrontal cortex and hippocampus were homogenized in PBS with $1 \mathrm{mM}$ phenylmethylsulfonyl fluoride (PMSF) and $1 \mathrm{mM}$ ethylene glycol tetraacetic acid (EGTA). Microtiter plates (48-well flat-bottom) were coated for $24 \mathrm{~h}$ with the samples diluted 1:2 in sample diluent and standard curves ranged between 7.8 and $500 \mathrm{pg} / \mathrm{ml}$. The plates were then washed four times with sample diluent and a monoclonal rabbit antibody diluted 1:1000 in sample diluent was added to each well. The plates were then incubated for $3 \mathrm{~h}$ at room temperature. After washing, a peroxidase-conjugated anti-rabbit antibody (diluted 1:1000) was added to each well and incubated at room temperature for $1 \mathrm{~h}$. After addition of streptavidin-enzyme, substrate and stop solution, the amount of IL- 6 was determined by absorbance at $450 \mathrm{~nm}$. The standard curve demonstrates a direct correlation between optical density (OD) and the concentration of IL-6. Total protein was measured by Lowry's method using bovine serum albumin as a standard.

Statistical analysis. Data are presented as mean \pm standard deviation (SD). Statistical analyses were performed by one-way analysis of variance (ANOVA). These statistical analyses were conducted by Statistical Product for Social Sciences (SPSS version 17.0$)$. $\mathrm{P}<0.05$ was considered to indicate a statistically significant result.

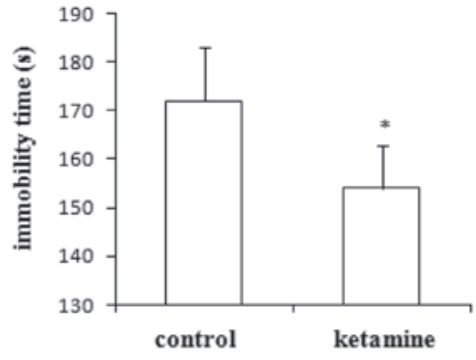

Figure 1. Effects of ketamine on the immobility time (mean $\pm \mathrm{SD}$ ) of rats during FST. Each group contained ten subjects. ${ }^{*} \mathrm{P}<0.05$, compared with the saline group. FST, forced swimming test.

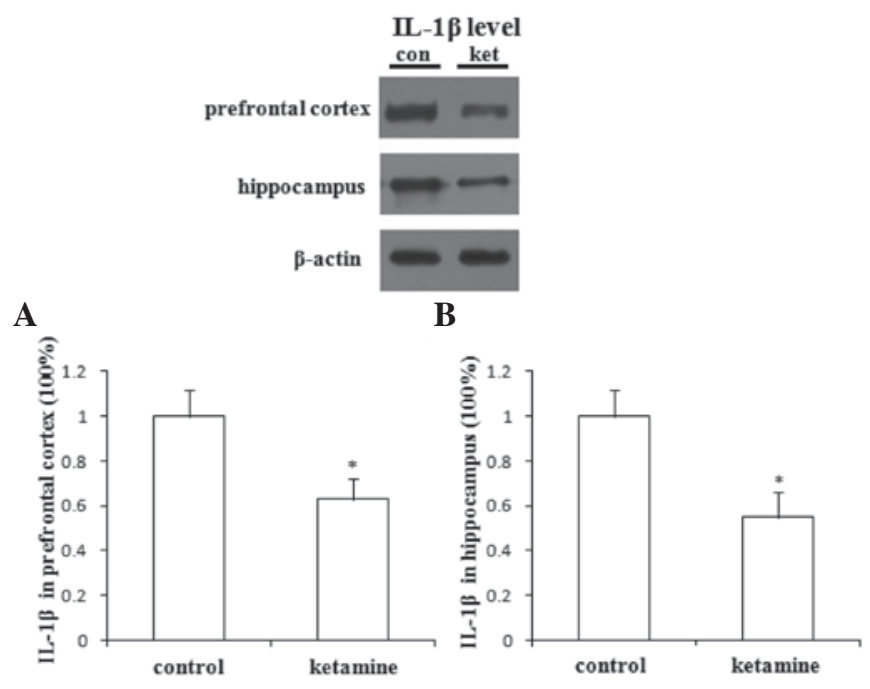

Figure 2. Effects of ketamine on the expression of IL-1 $\beta$ in rat (A) prefrontal cortex and (B) hippocampus. Each group contained ten subjects. ${ }^{*} \mathrm{P}<0.05$, compared with the saline group. IL, interleukin.
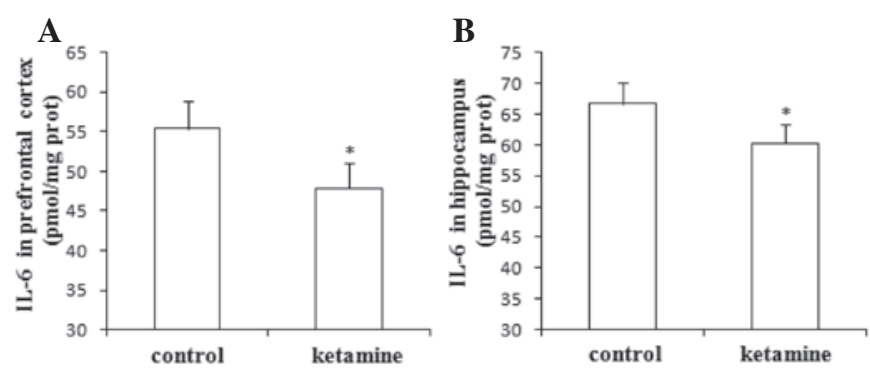

Figure 3. Effects of ketamine on the expression of IL-6 in rat (A) prefrontal cortex and (B) hippocampus. Each group contained ten subjects. " $\mathrm{P}<0.05$, compared with the saline group. IL, interleukin.

\section{Results}

Effects of ketamine on the immobility time of rats during FST. Compared with saline administration, the administration of ketamine significantly decreased the immobility time of rats during FST (P<0.05; Fig. 1).

Effects of ketamine on the expression of IL-1 $\beta$ in rat prefrontal cortex and hippocampus. Compared with saline administration, the administration of ketamine significantly decreased 
the expression of IL-1 $\beta$ in rat prefrontal cortex (Fig. 2A) and hippocampus (Fig. 2B; $\mathrm{P}<0.05$ ).

Effects of ketamine on the expression of IL-6 in rat prefrontal cortex and hippocampus. Compared with saline administration, the administration of ketamine significantly decreased the expression of IL-6 in rat prefrontal cortex (Fig. 3A) and hippocampus (Fig. 3B; $\mathrm{P}<0.05$ ).

\section{Discussion}

In the present study, we demonstrated that ketamine significantly decreased the immobility time of rats in FST, and there was a significantly lower expression of IL-1 $\beta$ and IL-6 in rat prefrontal cortex and hippocampus following ketamine administration.

Recent animal and clinical studies have indicated that the administration of ketamine at sub-anesthetic doses has fast-acting and robust antidepressant effects $(8,9)$, and it has been shown that ketamine, widely used as an anesthetic agent, demonstrates unique effects for the treatment of depression. In the present study, the administration of ketamine at a dose of $10 \mathrm{mg} / \mathrm{kg}$ significantly decreased the immobility time of rats receiving FST. This result is consistent with previous studies and confirmed ketamine's antidepressant effects.

It has previously been reported that ketamine is recommended for use in the anesthesia of patients with sepsis due to its effects of stimulating sympathetic nerves, maintaining vasoconstriction and maintaining circulation stability $(14,15)$. Taniguchi and Yamamoto (16) have indicated that ketamine may inhibit the sepsis-induced inflammatory response in a rat model and attenuate the fall of blood pressure. Furthermore, a previous study conducted by Sun et al (17) has revealed that ketamine's anti-inflammatory effects may be associated with the changes in the expression of nuclear factor- $\kappa \mathrm{B}$ and tumor necrosis factor- $\alpha$. Collectively, these findings suggest that ketamine has significant anti-inflammatory effects.

At present, the treatment of depression is mainly dependent on conventional antidepressant agents. A large body of evidence suggests that the peripheral serum proinflammatory cytokine levels of patients with depression are significantly higher than normal levels, and the increased levels are proportional to the severity of depression $(18,19)$. In addition, it has been demonstrated that the proinflammatory cytokine levels in the peripheral blood of patients with depression gradually tend to become normal following treatment with antidepressant agents $(20,21)$. In the preliminary study, we observed a lower expression of IL-1 $\beta$ and IL- 6 in rat prefrontal cortex and hippocampus after ketamine administration, and the results validated our above-mentioned hypothesis.

Mounting studies reveal that both IL-1 $\beta$ and IL- 6 are important in the etiology and pathophysiology of depression $(22,23)$. In clinical trials, elevated levels of IL-1 $\beta$ and IL-6 have been observed in the peripheral blood of depressed patients $(23,24)$. Besides, in experimental animals, administration of IL-1 $\beta$ has produced depressive-like symptoms which were attenuated by treatment with antidepressants $(25,26)$. Therefore, we concluded that the onset of antidepressants may be accompanied by the inhibition of IL-1 $\beta$ and IL- 6 . In the present study, the results demonstrated that ketamine exerts an antidepressant effect that is associated with the downregulation of IL-1 $\beta$ and Il-6 levels, which was consistent with the above-mentioned conclusion.

In conclusion, ketamine possesses properties that exert antidepressant effects, which have been confirmed in the present study, and its underlying mechanism is potentially associated with the inhibition of IL-1 $\beta$ and IL-6 expression in rat prefrontal cortex and hippocampus. However, a great limitation of the present study is that we did not test the levels of anti-inflammatory cytokines, which future studies are required to investigate further.

\section{Acknowledgements}

This study was supported by grants from the National Natural Science Foundation of China (Grant No. 30872424).

\section{References}

1. Kessler RC, Berglund P, Demler O, et al: The epidemiology of major depressive disorder: results from the National Comorbidity Survey Replication (NCS-R). JAMA 289: 3095-3105, 2003.

2. Berton $\mathrm{O}$ and Nestler EJ: New approaches to antidepressant drug discovery: beyond monoamines. Nat Rev Neurosci 7: 137-151, 2006.

3. Uppal A, Singh A, Gahtori P, Ghosh SK and Ahmad MZ: Antidepressants: current strategies and future opportunities. Curr Pharm Des 16: 4243-4253, 2010.

4. Mitchell AJ: Two-week delay in onset of action of antidepressants: new evidence. Br J Psychiatry 188: 105-106, 2006.

5. Wright CE, Strike PC, Brydon L and Steptoe A: Acute inflammation and negative mood: mediation by cytokine activation. Brain Behav Immun 19: 345-350, 2005.

6. Dantzer R: Cytokine-induced sickness behavior: mechanisms and implications. Ann NY Acad Sci 933: 222-234, 2001.

7. Pollak Y and Yirmiya R: Cytokine-induced changes in mood and behaviour: implications for 'depression due to a general medical condition', immunotherapy and antidepressive treatment. Int $\mathbf{J}$ Neuropsychopharmacol 5: 389-399, 2002.

8. Skolnick P, Popik P and Trullas R: Glutamate-based antidepressants: 20 years on. Trends Pharmacol Sci 30: 563-569, 2009.

9. Machado-Vieira R, Salvadore G, Diazgranados N and Zarate CA Jr: Ketamine and the next generation of antidepressants with a rapid onset of action. Pharmacol Ther 123: 143-150, 2009.

10. Dale O, Somogyi AA, Li Y, Sullivan T and Shavit Y: Does intraoperative ketamine attenuate inflammatory reactivity following surgery? A systematic review and meta-analysis. Anesth Analg 115: 934-943, 2012.

11. Ward JL, Harting MT, Cox CS Jr and Mercer DW: Effects of ketamine on endotoxin and traumatic brain injury induced cytokine production in the rat. J Trauma 70: 1471-1479, 2011.

12. Takahashi T, Kinoshita M, Shono S, et al: The effect of ketamine anesthesia on the immune function of mice with postoperative septicemia. Anesth Analg 111: 1051-1058, 2010.

13. Yang C, Hu YM, Zhou ZQ, Zhang GF and Yang JJ: Acute administration of ketamine in rats increases hippocampal BDNF and mTOR levels during forced swimming test. Ups J Med Sci: Sep 13, 2012 (Epub ahead of print).

14. Busch CJ, Spöhr FA, Motsch J, Gebhard MM, Martin EO and Weimann J: Effects of ketamine on hypoxic pulmonary vasoconstriction in the isolated perfused lungs of endotoxaemic mice. Eur J Anaesthesiol 27: 61-66, 2010.

15. Nakayama M and Murray PA. Ketamine preserves and propofol potentiates hypoxic pulmonary vasoconstriction compared with the conscious state in chronically instrumented dogs. Anesthesiology 91: 760-771, 1999.

16. Taniguchi $\mathrm{T}$ and Yamamoto K: Anti-inflammatory effects of intravenous anesthetics on endotoxemia. Mini Rev Med Chem 5: 241-245, 2005.

17. Sun J, Zhou ZQ, Lv R, Li WY and Xu JG: Ketamine inhibits LPS-induced calcium elevation and NF-kappa B activation in monocytes. Inflamm Res 53: 304-308, 2004. 
18. Raedler TJ: Inflammatory mechanisms in major depressive disorder. Curr Opin Psychiatry 24: 519-525, 2011.

19. Hannestad J, DellaGioia N and Bloch M: The effect of antidepressant medication treatment on serum levels of inflammatory cytokines: a meta-analysis. Neuropsychopharmacology 36 : 2452-2459, 2011

20. Catena-Dell'Osso M, Bellantuono C, Consoli G, Baroni S, Rotella F and Marazziti D: Inflammatory and neurodegenerative pathways in depression: a new avenue for antidepressant development? Curr Med Chem 18: 245-255, 2011.

21. Song $\mathrm{C}$ and Wang $\mathrm{H}$ : Cytokines mediated inflammation and decreased neurogenesis in animal models of depression. Prog Neuropsychopharmacol Biol Psychiatry 35: 760-768, 2011.

22. Owen BM, Eccleston D, Ferrier IN and Young AH: Raised levels of plasma interleukin-1beta in major and postviral depression. Acta Psychiatr Scand 103: 226-228, 2001.
23. Piletz JE, Halaris A, Iqbal O, et al: Pro-inflammatory biomakers in depression: treatment with venlafaxine. World J Biol Psychiatry 10: 313-323, 2009.

24. Brambilla F, Monteleone P and Maj M: Interleukin-1beta and tumor necrosis factor-alpha in children with major depressive disorder or dysthymia. J Affect Disord 78: 273-277, 2004.

25. Castanon N, Bluthe RM and Dantzer R: Chronic treatment with the atypical antidepressant tianeptine attenuates sickness behavior induced by peripheral but not central lipopolysaccharide and interleukin-1beta in the rat. Psychopharmacology (Berl) 154: 50-60, 2001

26. Merali Z, Brennan K, Brau P and Anisman H: Dissociating anorexia and anhedonia elicited by interleukin-1beta: antidepressant and gender effects on responding for 'free chow' and 'earned' sucrose intake. Psychopharmacology (Berl) 165: 413-418, 2003. 